\title{
La educación sigue siendo un "tesoro". Educación y docentes en los informes internacionales de la UNESCO
}

Roberto Sanz-Ponce y Aurelio González-Bertolín

\section{RESUMEN}

Los informes de la UNESCO (1973, 1996 y 2015) establecen las líneas centrales por las que debe transitar la educación en cada momento. En ellos se analiza la finalidad de la educación y el papel que juega el docente en la escuela. Por tanto, tiene un carácter prospectivo. El objetivo fundamental de este ensayo es desentrañar estos aspectos y enunciar algunos de los retos y desafíos a los que se va a enfrentar la educación y sus docentes en los próximos años, todo ello a la luz de las propuestas del organismo internacional.

Palabras clave: finalidad de la educación, UNESCO, docentes, educación.

Roberto Sanz-Ponce

roberto.sanz@ucv.es Español. Doctor por la Universidad de Valencia, España. Profesor de la Universidad Católica de Valencia, España. Director de la Revista Edetania. Estudios y propuestas socioeducativas y Secretario del Instituto Universitario de Teoría de la Educación, España. Temas de investigación: formación del profesorado, desarrollo de capacidades y resolución de conflictos en la escuela.

Aurelio González-Bertolín

aurelio.gonzalez@ucv.es

Español. Doctor por la Universidad de Valencia, España. Profesor de la Universidad Católica de Valencia, vicepresidente del Colegio Oficial de Pedagogos y Psicopedagogos de la Comunidad Valenciana y Secretario del Consejo de Colegios Oficiales de Pedagogos de España. Temas de investigación: formación del profesorado, historia de la educación y legislación educativa. 


\title{
A educação continua sendo um "tesouro". Educação e docentes nos informes internacionais da UNESCO
}

\section{RESUMO}

Os informes da UNESCO (1973, 1996 e 2015) estabelecem as linhas centrais pelas quais se deve transitar a educação em cada momento. Neles se analisa a finalidade da educação e o papel que joga o docente na escola. Por tanto, tem um caráter prospectivo. $\mathrm{O}$ objetivo fundamental deste ensaio é desentranhar estes aspectos e enunciar alguns dos desafios aos que vai enfrentar a educação e seus docentes nos próximos anos, tudo isso à luz das propostas do organismo internacional.

Palavras chave: finalidade da educação, UNESCO, docentes, educação.

\section{Education is still a "treasure". Education and teachers in the international reports of UNESCO}

\begin{abstract}
The UNESCO reports (1973, 1996 and 2015) establish the main lines through which education must move at all times. They analyze the purpose of education and the role played by the teacher in school. Therefore, it has a prospective nature. The main objective of this essay is to unravel these aspects and enunciate some of the challenges that will face education and its teachers in the coming years, all in light of the proposals that have been set out by the international organization.
\end{abstract}

Key words: purpose of education, UNESCO, teachers, education.

Recepción: 01/07/16. Aprobación: 29/03/17. 


\section{Introducción}

Veinte años han pasado desde la publicación del informe La educación encierra un tesoro, publicado por la Organización de las Naciones Unidas para la Educación, la Ciencia y la Cultura (Delors, 1996) y es momento - siempre lo es - de Repensar la educación. Cuando uno piensa sobre algún aspecto de la vida tiene una triple posibilidad de actuación. La primera es analizar ese aspecto mirando hacia el futuro, los nuevos retos y desafíos a los que se va a enfrentar y así poder establecer las líneas de intervención. La otra es mirar hacia el pasado y analizar lo vivido, las esperanzas que se tenían y todo aquello que no se hizo o no se pudo hacer. La tercera posibilidad es la combinación de ambas. Analizar el pasado para establecer las líneas de futuro. Ese es el objetivo de este artículo: ${ }^{1}$ confrontar las ideas que han ido surgiendo en los diferentes informes internacionales, con sus luces y sombras, frente a la realidad educativa actual, para ver qué queríamos conseguir y en qué estado nos encontramos. También estableceremos algunos aspectos que deberían repensarse en torno a la educación y analizaremos los desafíos de los docentes a partir de ahora.

Se trata de hacer un estudio acerca del concepto de Educación, en diferentes momentos y bajo distintos enfoques, atendiendo a las demandas que se le presentan a los docentes para acomodarse a las diferentes corrientes educativas, así como enunciar algunas líneas maestras que deberían ser atendidas por las facultades de Educación en la formación inicial de los futuros docentes. Y, todo ello a la luz de algunos informes de la UNESCO que por su relevancia y claridad se han convertido en referentes en el mundo educativo.

\section{La evolución del concepto de Educación a través de los Informes de la UNESCO}

Corría el año 1973 cuando veía la luz un documento de un gran calado educativo: Aprender a ser. La educación del futuro. Este informe ponía de relieve la importancia de la educación en el desarrollo de las personas y de los pueblos. Ya en el preámbulo definía a la educación "como problema de excepcional dificultad, y en todos los países sin excepción como tarea de la más alta importancia" (Faure, 1973: 25). A esta noble tarea debían dedicarse medios y esfuerzos de la sociedad civil, de los gobiernos y de las instituciones encargadas de la educación, ya que sólo así se podría conseguir una ciudadanía crítica y activa, que fomentase y defendiese la democracia, aún a sabiendas del peligro real que suponían algunos poderes públicos que se arrogaban en dueños de los sistemas educativos y los convertían en instrumentos de represión. En ese sentido, aquel momento histórico demandaba una ciudadanía instruida, que diesen respuesta a las secuelas que en las sociedades y mentalidades había ocasionado la II Guerra Mundial. Asimismo, esta nueva ciudadanía debía afrontar el cambio de paradigma económico mundial y luchar contra unas dramáticas y desmesuradas tasas de analfabetismo.

El acento de la educación se situaba, necesariamente, en la compleja tarea de alcanzar una efectiva escolarización universal en la enseñanza primaria o básica, poniéndose el foco en el aspecto cuantitativo de la educación — número de alumnos matriculados- - y no tanto en su variante cualitativa, es decir, la calidad del aprendizaje. Se proponía una educación que respondiese a una doble finalidad: por un lado, de carácter reproductor, que "concurre objetivamente a consolidar las estructuras existentes, a

\footnotetext{
${ }^{1}$ Este artículo se encuadra dentro del Proyecto "Evaluación de la calidad del profesorado en universidades de México y España", en el que participan siete universidades mexicanas y cuatro españolas. Este Proyecto ha sido financiado por la Convocatoria de Redes Temáticas de Colaboración Académica 2015 del Programa para el Desarrollo Profesional Docente en Educación Superior (PRODEP) de la Secretaría de Educación Pública (SEP) de México.
} 
formar individuos aptos para vivir en la sociedad tal y como es" (Faure, 1973: 117) y, por tanto, con una visión conservadora en el buen sentido del término. Y, por otro, se apostaba por una educación renovadora, que transformase los cimientos de la sociedad y la modernizase, que cambiase las estructuras y los roles educativos, tomando como referencia la figura del alumnado, convirtiéndolo en el actor principal del proceso educativo. En definitiva, se trataba de racionalizar y humanizar la enseñanza, sin olvidar que "la escuela debe estar hecha para el niño, y no el niño para la escuela" (Faure, 1973: 120).

En ese sentido, no debemos soslayar el estado en el que se encontraban las escuelas y la propia educación. El Informe reconoce que la escuela de los años setenta se caracterizaba por sus "injusticias, discriminaciones,...", acusación recurrente sobre la escuela, por más que a muchos les pareciera sorprendente que se le reprochase "ser como ha sido hecha a través de los siglos, por los mandatos sucesivos que ha recibido de la sociedad, y a la que todos, por diferentes que pudieran ser, estaban de acuerdo en exigir que fuese autoritario y jerárquico" (Faure, 1973: 132).

Contra esta visión tan negativa de la escuela se exige una profunda transformación, que la convierta en una compensadora de desigualdades sociales y económicas, instaurando un sistema democrático, abierto y sin barreras sociales, preocupada por el descubrimiento del conocimiento. Y todo ello, atendiendo al principio de igualdad, que "no consiste, como en general todavía se cree, en garantizar un trato idéntico a todos, en nombre de una igualdad formal, sino en ofrecer a cada individuo un método, un ritmo y unas formas de enseñanza que le acomoden" (Faure, 1973: 139). Se trata, por tanto, de fomentar el "Aprender a vivir" y el "Aprender a aprender", el pensamiento crítico y libre, el aprender a amar el mundo y hacerlo más humano; pero fundamentalmente, se trata de potenciar el componente personal de la educación (Aprender a ser), en un intento de unir al Homo Sapiens y al Homo Faber y ponerlo en armonía consigo mismo y con los demás, con el Homo Concors.

Y así llegamos a 1996 y la UNESCO publica su segundo gran Informe: La educación encierra un tesoro. En este momento el objetivo fundamental se centraba en educar para una sociedad mundial: “¿Cómo aprender a vivir juntos en la 'aldea planetaria' si no podemos vivir en las comunidades a las que pertenecemos por naturaleza: la nación, la región, la ciudad, el pueblo, la vecindad?” (Delors, 1996: 16), es decir, si queremos y somos capaces de participar en la vida en comunidad. Para ello, la educación debía hacer consciente al individuo de sus raíces, de su singularidad; y al mismo tiempo enseñarle a respetar a las otras culturas, hacerle conocedor de la existencia de un patrimonio común de la humanidad. Por tanto, se responsabilizaba a la escuela de la edificación de un mundo más solidario y humano, convirtiéndola en un instrumento de "cohesión social", de atención a la diversidad y de lucha frente a la exclusión social.

Además, añadía que la conformación de una sociedad mundial e intercultural, en la que se permitiese desarrollar los talentos y capacidades de todos en aras de realizar un proyecto personal y profesional de vida, es una tarea conjunta y compartida entre la escuela, la familia y las comunidades locales. La educación también supone una experiencia social, en la que el niño/a va conociéndose a sí mismo/a al tiempo que enriquece sus relaciones con los demás. "Esta experiencia debe iniciarse antes de la edad escolar obligatoria según diferentes formas en función de la situación, pero las familias y las comunidades locales deben involucrarse" (Delors, 1996: 25), por lo que es necesario especificar las tareas y las funciones de cada una de las instituciones involucradas.

Un año antes, en 1995, el Documento de Política para el Cambio y el Desarrollo en la Educación Superior se planteaba dar respuesta a cuál es y cuál debiera ser la función de la educación superior en la sociedad. Se trataba de buscar el difícil equilibrio 
entre la educación entendida como bien público, es decir, como el pleno desarrollo de las capacidades y la personalidad de los estudiantes y una formación superior más orientada a la empleabilidad de los educandos. Por tanto, se concluye que el apoyo público a la educación superior "sigue siendo esencial para que esta pueda desarrollar su misión educativa, social e institucional" (UNESCO, 1995: 34).

El mundo, en aquellos años, a los ojos de los responsables de la UNESCO, se enfrentaba a una serie de problemas y tensiones en torno a los siguientes conflictos: agravación de las desigualdades sociales, desarraigo provocado por las migraciones y una profunda crisis moral mundial, con consecuencias palpables en el aumento de la violencia y de la delincuencia. Además, afirma el Informe Delors, la escuela tampoco había cumplido con los objetivos planteados en el Informe anterior Aprender a ser. La fotografía que presentan no es nada halagüeña: fracaso escolar, violencia en las aulas, discriminaciones..., muchas veces la escuela - continúa diciendo - se convertía en el foco del problema y no en la solución, ejerciendo como un factor de exclusión social. Este fracaso educativo afectaba con mucha más virulencia a los jóvenes de medios y familias desfavorecidas, siendo esto "en cualquier caso una catástrofe, absolutamente desoladora en el plano moral, humano y social, que muy a menudo genera exclusiones que marcarán a los jóvenes durante toda su vida de adultos" (Delors, 1996: 156).

Por todo ello, se plantea la necesidad de luchar contra el fracaso escolar como un imperativo de orden social, con base en dos medidas fundamentales: personalizar la enseñanza y devolver al sistema educativo a los excluidos. Además, se concibe como urgente la preparación, desde la escuela, para la participación activa en la "cosa pública", la "alfabetización política" (Delors, 1996: 65), criticándose una enseñanza excesivamente preocupada por la productividad y el crecimiento económico. "Ya no es posible pedir a los sistemas educativos que formen mano de obra para un empleo industrial estable; se trata más bien de formar para la innovación personas capaces de evolucionar, de adaptarse a un mundo en rápida mutación" (Delors, 1996: 78). Por tanto, se trata de alejar y superar una concepción utilitarista y al servicio del mercado, para acercarse a una educación de carácter humanista, preocupada por la persona, por sus talentos y aptitudes, "actualizando así el tesoro escondido en cada uno de nosotros" (Delors, 1996: 96), que respeta y cuida el medio ambiente y la diversidad de tradiciones y de culturas.

Y todo ello, atendiendo a una de las mayores aportaciones del Informe al mundo educativo: los cuatro pilares de la educación para el siglo XXI: Aprender a Conocer, Aprender a Hacer, Aprender a vivir juntos/Aprender a vivir con los demás y Aprender a Ser.

El tercer gran informe de la UNESCO se publica en 2015, con el título: Repensar la educación. ¿Hacia un bien común universal? Este Informe se plantea como una reflexión todavía necesaria acerca de la educación, tomando como punto de partida las dos publicaciones anteriores: Aprender a ser: la educación del futuro (Faure, 1973) y La educación encierra un tesoro (Delors, 1996) y los resultados obtenidos, con sus avances y retrocesos, en todos estos años.

A diferencia del enfoque que se hacía en torno a la educación en 1972, cuando aparece Aprender a ser, el nuevo Informe se centra en el aspecto cualitativo de la enseñanza, la calidad del aprendizaje y su verdadera efectividad y no tanto ya, aunque sin olvidarlo, en el derecho de acceso a la educación. Se trata de ir más allá de las competencias básicas numéricas y lingüísticas, para "centrarse en los entornos de aprendizaje y en nuevos enfoques del aprendizaje que propicien una mayor justicia, la equidad social y la solidaridad mundial" (Bokova, 2015a: 3).

Nuevamente, al igual que en los anteriores informes, se trata de dotar de una visión humanista a la educación aunque, tal vez conociendo los resultados pasados, con mucha menos convicción. La pregunta planteada en el título del Informe no oculta las dudas 
acerca del éxito de la empresa iniciada: " ¿Hacia un bien común mundial?", interpretando la dificultad del acometido y la multitud de obstáculos a los que enfrentarse. Este planteamiento humanista se preocupa "ante todo por la inclusión y por una educación que no excluya ni margine" (Bokova, 2015a: 37) a ningún niño/a o joven en riesgo evidente de exclusión social. "Todo esto tiene consecuencias importantes en la concepción y aplicación de políticas de educación que sean sensibles a los conflictos. Éstas han de ser inclusivas [...], si no queremos que la educación se limite a reproducir desigualdades y tensiones sociales" (Bokova, 2015a: 25).

En el año del informe (2015), aquella situación de desigualdad social que denunciaban los otros informes y a cuyo problema pretendían poner remedio, se ve agravada a causa de la globalización económica, aumentando las diferencias entre los distintos países y en cada uno de ellos. Entre los datos que se manejan, se afirma que: "la riqueza del mundo se divide así: casi la mitad pertenece al $1 \%$, y la otra mitad al 99\% restante. Esta desigualdad en los ingresos $[. .$.$] contribuye a fomentar la exclusión social$ y a socavar la cohesión de la sociedad" (Bokova, 2015a: 23).

Como es obvio, la educación no ha sido capaz de solucionar esta problemática, incluso en ocasiones ha contribuido a su crecimiento, instaurando dos tipos de educación en función de las rentas del alumnado. El alumnado con menos recursos y con más necesidades suele tener menos posibilidades de promoción, que el alumnado con más recursos y posibilidades. Por tanto, la escuela no ha tomado, en todos los casos, tal y como se pedía en los Informes, el rol de compensadora de desigualdades. Por ello, el Informe pretende superar el carácter estrictamente utilitario del discurso internacional del desarrollo. "La educación no conlleva únicamente la adquisición de aptitudes, sino también la de los valores de respeto a la vida y a la dignidad humana necesarios para que reine la armonía social en un mundo caracterizado por la diversidad" (Bokova, 2015a: 37). Por tanto, se reclama mayor justicia, menor brecha social, mayores derechos humanos para todos y menos personas en situación de "in-dignidad".

Ante esta situación, nada nueva por otra parte, la educación se plantea como finalidad el "desarrollo humano y social", pero añade un ingrediente adicional: "sostenible". El Informe define la sostenibilidad como la acción responsable, tanto de individuos como de sociedades, en aras de "un futuro mejor para todos, a nivel local y mundial, un futuro en el que el desarrollo socioeconómico responde a los imperativos de la justicia social y la gestión ambiental" (Bokova, 2015a: 20). Una recurrente visión del desarrollo únicamente centrada en la productividad y en las leyes del mercado nos han conducido a problemas de sobreexplotación y de cambio climático, por lo que se ha dado un giro inesperado a los fines de la educación: el cuidado del medio ambiente.

La educación, en ese sentido, se convierte en un factor esencial para favorecer el uso de fuentes alternativas renovables y para atenuar el impacto del cambio climático, así como para preparar a las nuevas generaciones para una realidad ecológica, social y económica cambiante. Por ello afirma el Informe que "una auténtica educación es aquella que forma los recursos humanos que necesitamos para ser productivos, seguir aprendiendo, resolver problemas, ser creativos y vivir juntos y con la naturaleza en paz y armonía” (Bokova, 2015a: 32).

Bajo esta definición la educación adquiere una importancia radical en la formación del ser humano y en el cuidado del medio ambiente, convirtiéndose en el "motor del desarrollo sostenible y la clave de un mundo mejor" (Bokova, 2015a: 32), dotando a las personas de las capacidades necesarias para "vivir una vida con sentido y con igual dignidad" (Bokova, 2015a: 33), alejándose de cualquier propuesta que aliene al individuo y lo trate como una mera mercancía o que imponga prácticas sociales que dividen y deshumanizan a las personas. 


\section{El papel de los docentes en los diferentes informes de la UNESCO}

En 1973, ya comenzaba a vislumbrarse un nuevo rol a la figura docente en la escuela, en el que se pasaba de un docente transmisor de conocimientos a otro de talante más innovador y reflexivo. La facilidad para acceder a una mayor cantidad de información modificaba sustancialmente su papel. Éste ya no era la única vía de acceso a esta información, ni seguramente la más efectiva y completa, por lo que su labor se dirigía a valorar, clasificar, discernir..., así como a fomentar el pensamiento crítico y autónomo de los estudiantes y su educación en valores. "El enseñante, al lado de sus tareas tradicionales, está llamado a convertirse cada día más en un consejero, un interlocutor; más bien la persona que ayuda a buscar en común los argumentos contradictorios, que la que posee las verdades prefabricadas" (Faure, 1973: 142).

Por ello, a los docentes se les debía pedir algo distinto a lo que venían haciendo y que tenía mucha relación con el Aprender a vivir, Aprender a aprender a lo largo de toda la vida, Aprender a pensar, Aprender a amar el mundo y humanizarlo, y Aprender a ser, creativos. Y en esta nueva realidad, el acto de enseñar deja paso al acto de aprender. Hace al objeto de la educación (alumno) el sujeto de su propia educación. "Ya no recibe la educación como un don $[\ldots]$; se asimila al precio de una conquista sobre el saber y sobre sí mismo que le convierte en el dueño, no en el recipiente, de los conocimientos que adquiere" (Faure, 1973: 241).

Ahondando en esa misma dirección, el Informe Delors remarcaba el sentido, con nuevos matices, de la misión destinada al docente. "Es deseable que la escuela le inculque más el gusto y el placer de aprender, la capacidad de aprender a aprender, la curiosidad del intelecto" (Delors, 1996: 21). Ésta se centra más en encender la llama de la curiosidad y del aprendizaje y, no tanto, en la tradicional labor de llenar el vaso. A los docentes corresponde un papel determinante en la formación de las actitudes de sus alumnos/as con respecto al aprendizaje. "Ellos son los que deben despertar la curiosidad, desarrollar la autonomía, fomentar el rigor intelectual y crear las condiciones necesarias para el éxito de la enseñanza formal y la educación permanente" (Delors, 1996: 161).

Los docentes se enfrentaban a la difícil misión de hacer más atractiva la escuela y de preparar al alumnado para el acceso a la sociedad del conocimiento. Se convierten en "agentes de cambio" hacia una sociedad más justa y democrática, y por ello su formación y capacidad comienzan a relacionarse con la calidad de la educación. El Informe aboga por revisar los mecanismos de contratación docente, mejorar la formación inicial y continua, y las condiciones de trabajo y reconocimiento social como estrategias para mejorar la calidad de los sistemas educativos. Y se plantea: “Qué puede esperar razonablemente la sociedad del personal docente? [...] ¿Quién puede ser un buen maestro o profesor, y cómo encontrar a esa persona, cómo formarla y cómo preservar su motivación y la calidad de su enseñanza?" (Delors, 1996: 162).

Además, matiza algunas cuestiones de obligado cumplimiento para los nuevos enseñantes.

1. Se les debe considerar como agentes de cambio, como referentes para inculcar el gusto y el placer de aprender. Se les exige la capacidad de motivación, de convertirse en un generador del deseo de aprender, investigar y descubrir.

2. Se les exige la capacidad de formar generaciones críticas, libres, responsables y capaces de tomar las riendas de su vida para vivirla de manera plena y digna. "El aporte de maestros y profesores es esencial para preparar a los jóvenes, no sólo para que aborden el porvenir con confianza, sino para que ellos mismos lo edifiquen de manera resuelta y responsable" (Delors, 1996: 161).

3. Les corresponde la formación de las actitudes, tanto positivas como negativas, del alumnado frente al estudio. "Ellos son los que deben 
despertar la curiosidad, desarrollar la autonomía, fomentar el rigor intelectual y crear las condiciones necesarias para el éxito de la enseñanza formal y la educación permanente" (Delors, 1996: 161). Se les demanda profesionalidad en la tarea educativa: vocación, actitudes y comportamientos positivos hacia el conocimiento. El ejemplo de la formación continua y la búsqueda incansable del conocimiento son el motor del deseo, del rigor y de la perseverancia en el trabajo y en el estudio de los estudiantes.

4. "Debe establecer una nueva relación con el alumno, pasar de la función de 'solista' a la de 'acompañante', convirtiéndose ya no tanto en el que imparte los conocimientos como en el que ayuda a los alumnos a encontrar, organizar y manejar esos conocimientos" (Delors, 1996: 164). Ser flexible en cuanto a los métodos y ritmos de enseñanza, pero firmes en la educación de los valores morales.

5. Se les exige replantearse la manera de enseñar. Dejar de ser un transmisor de respuestas, cerradas y únicas, para convertirse en un potenciador de preguntas, de dudas. Lanzar interrogantes, plantear problemas y guiar y orientar con sus respuestas y soluciones. Dejando al alumno/a que piense, que busque, que razone, en definitiva, que aprenda y que crezca como persona.

6. Los docentes se convierten en modelos de comportamiento y de vida, por lo que se les reclama coherencia entre su discurso y sus valores; honestidad en sus actos; humildad en su proceder, admitiendo sus errores y estando dispuestos a aprender en cualquier momento. Ser referentes morales, intelectuales, sociales... "La gran fuerza de los docentes es la del ejemplo" (Delors, 1996: 167).

Por último, Repensar la educación (Bokova, 2015a), sitúa al docente en la encrucijada de educar a las nuevas generaciones en la difícil sociedad del conocimiento. Existen voces que alertan del peligro de la desaparición de la figura del docente, aunque, desde la UNESCO, se afirma que tan sólo se trata de un cambio de orientación en sus funciones. "El docente pasa a ser un guía que permite a los estudiantes, desde la primera infancia y durante toda la trayectoria de su aprendizaje, desarrollarse y avanzar en el laberinto cada vez más intrincado del conocimiento" (Bokova, 2015a: 57).

Asimismo, las tareas del docente tenderán a replantearse y remodelarse continuamente en función de las exigencias y desafios que se vayan planteando en un mundo globalizado y en constante transformación. Por ello se hace necesario implantar una formación inicial y continua que capacite al profesorado para adaptarse a las nuevas situaciones, pero al mismo tiempo se les debe ofrecer unas condiciones de vida y laborales acordes a esta formación e inestabilidad, condiciones atractivas y motivantes con el objetivo de atraer a los mejores estudiantes a la profesión del Magisterio.

\section{Los retos y desafíos de la educación y sus docentes en la escuela del siglo $\mathrm{XXI}$}

\section{Los nuevos retos de la educación}

Uno de los retos más importantes a los que se enfrenta la educación es garantizar el derecho a la educación de todos los niños/as y jóvenes del mundo en edad escolar. El derecho de acceso, en igualdad de condiciones, no queda reconocido en todos los países y esto constituye un desafio fundamental.

Los informes de Seguimiento de la Educación para Todos (Matsuura, 2008; Bokova, 2012; Bokova, 2015b) remarcan la gravedad del abandono escolar temprano (UNESCO, 2012) en muchos países del mundo. Además de este abandono temprano también analizan y denuncian, con preocupación, los resultados de aprendizaje y aprovechamiento escolar de los alumnos/as en las escuelas.

En ese sentido, tanto los informes analizados de la UNESCO como otros informes menores de 
instituciones internacionales, ponen el acento del derecho a la educación en la calidad del aprendizaje, singularmente en aquellos países donde ya queda reconocido el derecho de acceso. En 2013, la UNESCO y el Centro de Educación Universal de Brookings, publicaban Hacia un aprendizaje universal. Lo que cada niño debería aprender (UNESCO-CUE, 2013a) y afirmaban que para que todos los beneficios personales y sociales que se le reconocen a la educación sean reales, los alumnos/as deberían aprender mientras se encontrasen en la escuela: "El derecho humano a la educación no puede materializarse simplemente al garantizar que los niños asistan a la escuela; ellos deben además aprender mientras se encuentran allí" (UNESCOCUE, 2013a: 10). Meses más tarde, esta misma Comisión sacaba a la luz, Hacia un aprendizaje universal. Recomendaciones de la comisión especial sobre métricas de los aprendizajes, donde era, si cabe, más explícito: "Está demostrado que son los niveles de aprendizaje y no los años pasados en la escuela lo que produce rendimientos sobre la inversión en educación respecto a lo social y económico, incluyendo empleo, productividad y crecimiento" (UNESCO-CUE, 2013b: 9).

Estas reflexiones en torno al aprendizaje real y efectivo de los estudiantes plantean dos nuevos retos a la educación del siglo XXI: ¿qué hacemos con aquellos alumnos/as que fracasan en la escuela? y ¿cuáles deberían ser los contenidos básicos - competencias o dominios de aprendizaje - que todos deberían adquirir para desenvolverse en su vida de una manera digna y plena? Ambas reflexiones son, sin duda alguna, dos de los temas que deben ser abordados en los próximos años por los expertos educativos, organismos internacionales y gobiernos nacionales, para garantizar una educación de calidad, respetar el derecho a la educación y fomentar la sostenibilidad económica y medio ambiental.

El segundo gran reto al que se enfrenta la educación es el de humanizar la escuela, los procesos educativos y los resultados que se derivan de los aprendizajes. Desde hace tiempo, los informes del
Programa de Naciones Unidas para el Desarrollo (PNUD) establecen las bases de una educación más centrada en la persona y su desarrollo. El desarrollo humano frente al desarrollo del Producto Interior Bruto (PIB) constituye una de las reivindicaciones de Amartya Sen, Sakiko Fukuda y sus respectivos equipos (Nussbaum y Sen, 1996). En sus Informes se viene afirmando, reiteradamente, la idea de que la riqueza de cualquier país se encuentra en su gente (PNUD, 1999, 2000 y 2004) y no tanto en los ingresos y recursos naturales o materiales que posea.

Nussbaum da un paso más en la defensa de una educación centrada en las personas. Con sus escritos trata de desmitificar esa concepción educativa preocupada sólo por un desarrollo económico, y no por la calidad de vida de todos sus ciudadanos/as. Nos habla de la llegada de una "crisis silenciosa" en materia educativa, que producirá "generaciones enteras de máquinas utilitarias, en lugar de ciudadanos cabales con la capacidad de pensar por sí mismos, poseer una mirada crítica sobre las tradiciones y comprender la importancia de los logros y los sufrimientos ajenos" (Nussbaum, 2010: 20). Y de esta manera, va perfilando la teoría del desarrollo humano como desarrollo de capacidades (Nussbaum, 2013: 16), como una "contrateoría que necesitamos en esta era de problemas humanos acuciantes y de desigualdades injustificables."

En este intento por humanizar la educación, por alejarla de una visión mercantilista, se necesita de un replanteamiento de los contenidos, capacidades y competencias que deben adquirirse en la escuela. Este Repensar la educación tiene que fijar como tarea prioritaria responder a la finalidad de la educación. Perrenoud (2012) afirma que se debe dar un giro radical al sentido de la educación y preparar a los niños/as y jóvenes para la vida. Esta visión no es al 100\% compartida por organizaciones como la Organización para la Cooperación y el Desarrollo Económico (OCDE) (2015), que en uno de sus últimos documentos aboga por la revisión de los curriculum 
académicos, pero con miras a introducir competencias para responder a las necesidades del mercado laboral. Tampoco olvidemos que los contenidos escolares se han planteado históricamente como base para continuar nuevos estudios, es decir, con un carácter propedéutico; y no para preparar a todos para poder desarrollar una vida digna. Además, afirma que "aquellos a los que la escuela debería preparar mejor para la vida son los que saldrán del sistema educativo sin haber adquirido el nivel de cultura suficiente como para aprender fácilmente en la edad adulta lo que no habrán aprendido en la escuela obligatoria" (Perrenoud, 2012: 29).

Probamente este planteamiento pueda ayudar a combatir una de las grandes lacras del siglo XXI: la desigualdad social entre las personas (Bokova, 2012 y 2015b; Eurydice, 2009; Matsuura, 2008; OEI, 2014). La educación y la escuela deben favorecer la erradicación de estas desigualdades y tal como manifiestan los grandes informes de la UNESCO, hasta el momento han sido incapaces. Preparando para la vida se podrían compensar los déficits personales de los grupos más desfavorecidos para enfrentarse a la vida con dignidad. En esa misma línea, la OCDE (2015: 50) mantiene que "entre los principales [retos] se encuentra apoyar y aumentar el éxito y nivel de los estudiantes de bajo rendimiento, de los estudiantes de entornos socioeconómicos desfavorables y de los subgrupos de población (como inmigrantes o minorías étnicas), y reducir el impacto del entorno socioeconómico en los resultados educativos".

Otro de los grandes retos a los que se enfrenta la educación es el dilema entre lo local y lo universal. Desde la publicación del Informe Delors (1996) se plantea la necesidad de educar para vivir y convivir en la gran "aldea planetaria", bajo el pilar de Aprender a vivir juntos/Aprender a vivir con los demás. La escuela debe perseguir el equilibrio entre una educación que enfatice la singularidad, la unicidad de cada persona y, al mismo tiempo, que muestre todo aquello que nos une, que nos convierte en "ciudadanos del mundo".
Nadie duda de la necesidad de dotar de raíces a la persona a través de la enseñanza, arraigarla con lo más cercano, con el terruño y con la gente con la que convive y comparte una cultura propia, unos valores y una historia común. Pero al mismo tiempo, gracias al proceso de globalización, que ha derribado las fronteras y ha transformado el mundo en un lugar sin distancias, se debe educar a las personas en una "pedagogía cosmopolita," tal y como la definía Horace Mann, dotándolas de los valores propios de una "ciudadanía universal". Es cierto que estas políticas educativas tienen el riesgo de reducir la diversidad e ir poco a poco limando las diferencias culturales propias de cada lugar, conformando una ciudadanía estandarizada. Pero, como nos interpela el profesor Escámez (2013: 19): “'Se les debe enseñar a los estudiantes que, por encima de todo, son ciudadanos de una nacionalidad, Estado o comunidad política de Estados, o más bien debería enseñárseles que, más allá de eso, son ciudadanos de un mundo de seres humanos?" Por lo tanto, aun cuando nos encontremos en un estado concreto, debemos tener la capacidad y la actitud de compartir el mundo con la ciudadanía de otros estados. Nussbaum (1999), en esa misma línea, afirma que se precisa de cierta lealtad hacia lo común de la humanidad, hacia ese parentesco universal entre todos los hombres.

En este sentido, ¿puede la escuela permitir una educación de sus jóvenes que olvide a aquellas personas que no tengan los medios, recursos y posibilidades de desarrollar sus capacidades básicas y sus derechos humanos? ¿Puede permitir la existencia de personas que viven por debajo del umbral de la dignidad humana en la búsqueda de una sociedad pacífica, tolerante, solidaria, respetuosa, sostenible y humana, tal y como reclaman insistentemente todos los informes de la UNESCO sobre educación? Tal vez, tenga razón el último Informe publicado por la UNESCO cuando reclama Repensar la educación y se plantea la pregunta: ¿Hacia un bien común universal?, reclamando un giro radical en las políticas educativas 
y en las acciones docentes. "De otro modo, no hacemos sino educar una nación de hipócritas morales que hablan el lenguaje del universalismo pero cuyo universo, por el contrario, tiene un alcance restringido e interesado" (Nussbaum, 1999: 24).

El último gran reto al que debe hacer frente la educación es el desarrollo humano, ecológico, social y económico sostenible. El mundo se enfrenta a una serie de modelos económicos, tecnológicos, humanos y medioambientales que son totalmente insostenibles y a los que la educación debe dar respuesta de manera urgente.

El modelo económico nacido de la globalización ha traído consigo la reducción de los índices de pobreza en el mundo en términos generales, aunque también algunas consecuencias dramáticas: subempleo, desempleo juvenil, trabajo precario..., lo cual ha provocado, en algunos lugares, tensiones sociales, inestabilidad política y conflictos violentos, aumentando la vulnerabilidad, desigualdad, exclusión y violencia entre muchas personas del mundo.

Este modelo económico también se sustenta sobre modelos de producción y consumo insostenible. El uso excesivo - abuso - de los recursos naturales no renovables y la contaminación medioambiental provocan el calentamiento del planeta, el deterioro del medio ambiente, el recrudecimiento de las catástrofes naturales; en definitiva, el cambio climático. El crecimiento demográfico y la urbanización también son consecuencia de este nuevo modelo económico. La población mundial se ha triplicado en los últimos 65 años, concentrándose un porcentaje altísimo en los núcleos urbanos. Este aumento demográfico también provoca el aumento del consumo de los recursos naturales (agua, alimentos, energía, madera...) agravando el problema. Todo ello conduce al planeta a un "estrés ecológico".

Estas circunstancias económicas no sólo tienen consecuencias para el medio ambiente, sino que fundamentalmente afectan a las personas que lo habitan. El medio ambiente y el ser humano se degradan conjuntamente. Problemas de superpoblación, de escasez o agotamiento de recursos, contaminación, desigualdad social, pobreza extrema, escasez de agua y alimentos..., afectan a personas que viven en los límites de la dignidad humana.

$\mathrm{Y}$ en ese contexto, la educación adquiere una misión prioritaria en la búsqueda de un desarrollo humano, social, económico y ecológico sostenible. En primer lugar, haciendo conscientes a los jóvenes de las causas y consecuencias; en segundo lugar, sensibilizándolos y, por último, cambiando "de forma significativa las pautas actuales de desarrollo, producción, consumo y comportamiento" (Aznar y Ull, 2013: 12; Francisco I, 2015). Y esta concienciación, sensibilización y cambio conductual deben tener consecuencias en las personas, mejorando su calidad de vida, repartiendo de manera equitativa los bienes y recursos, cubriendo las necesidades básicas de toda la población y reconociendo y respetando los derechos humanos. En ese sentido, la Declaración del Milenio (ONU, 2015) remarcaba entre sus ocho objetivos para el milenio, cuatro que hacen referencia expresa a estos asuntos, y la educación debe necesariamente articularse como el motor de este cambio.

\section{Los docentes ante nuevos desafíos}

El factor más determinante para la calidad de la educación es, sin duda alguna, mejorar la formación y las condiciones laborales del profesorado (Barber y Mourshed, 2008). Éste se enfrenta a una serie de nuevos desafíos, que no sólo responderán a las demandas pedagógicas propias del proceso de enseñanza-aprendizaje, sino que fundamentalmente vendrán descritas en función del rol que se le asigne a la escuela misma (OCDE, 2001; Comisión de las Comunidades Europeas, 2007). La escuela actual se enfrenta a la pérdida del monopolio en cuanto a la promoción del conocimiento; a la globalización económica, política y cultural que se opone a una escuela de tipo local, y a las nuevas formas de aprendizaje o autoaprendizaje a través de herramientas informáticas, que desplazan o reducen el impacto de 
las escuelas y de sus docentes. Para Fullan (2002), la capacidad del profesorado para enfrentarse al cambio y aprender de las nuevas demandas será clave para el desarrollo de las sociedades y de las personas, aunque actualmente "no están en posición de desempeñar este vital papel. Necesitamos una nueva mentalidad que permita a los educadores convertirse en agentes, y no en víctimas, del cambio" (Fullan, 2002: 9). Esto conllevará el replanteamiento de las competencias del profesorado y de las políticas educativas para adaptarse a las nuevas demandas sociales (Martín et al., 2001).

El primer gran desafío al que se enfrentan los docentes hace referencia a su capacidad para desarrollar los talentos de los alumnos. Hargreaves (2000) definía al profesor como un agente de cambio cualificado, que investiga en el aula y que trabaja de manera conjunta con otros compañeros/as en busca del aprendizaje de sus estudiantes. Esta investigación en el aula, en la acción educativa (Stenhouse, 1988), supone adaptar las características de la enseñanza a las peculiaridades del alumnado, a sus formas distintas de aprender, a sus diferentes ritmos y a las diversas capacidades de estos. Por tanto, se trata de un profundo cambio de modelo, en el que el centro del proceso educativo se sitúa en el alumno/a, que se convierte en un agente activo, que precisa de metodologías participativas y experienciales: tareas, aprendizaje basado en problemas o aprendizaje basado en retos (Observatorio de Innovación Educativa del Tecnológico de Monterrey, 2015).

El docente adquiere el rol de mentor, de orientador, de experto en la creación de situaciones de aprendizaje, que fomenta la creatividad, la motivación por el aprendizaje, por el descubrimiento de lo desconocido y por la innovación. En esa misma línea, la OCDEUNESCO (2001) demandaban a los docentes capacidad pedagógica, motivación para el aprendizaje, creatividad y cooperación. Además, el docente se enfrenta a nuevos desafios a la hora de planificar y estructurar sus enseñanzas. Dos importantes investigaciones realizadas con docentes remarcan la importancia de construir conocimiento junto al alumnado, generar pensamiento autónomo y crítico, mediante situaciones problema y preguntas para la reflexión. Para ello, estos deben dominar perfectam ente la materia que imparten y poseer multitud de recursos didáctico-metodológicos (Bain, 2007 y Finkel, 2008).

En ese sentido, los avances de la neuropedagogía señalan el camino a recorrer, las posibilidades de actuación y lo mucho que nos queda por aprender. La OCDE publicaba La comprensión del cerebro. El nacimiento de una ciencia del aprendizaje, y describía las grandes esperanzas que se abrían para un futuro cercano en el mundo educativo: "'comprender el cerebro' puede ayudar a abrir nuevos caminos para mejorar la investigación, las políticas y las prácticas en el campo educacional" (OCDE, 2007: 19), si bien alertan de que estos descubrimientos no son la panacea ni la varita mágica para asegurar el aprendizaje (Pizarro, 2003; Blakemore y Frith, 2005; Forés y Ligioiz, 2009; Howard-Jones, 2011; Mora, 2015). Nos describen una serie de características que favorecen el aprendizaje: el conocimiento de los "periodos sensibles" o ventanas de oportunidad, como momentos óptimos para el aprendizaje; la plasticidad del cerebro a lo largo de toda la vida; la desmitificación de ciertas premisas erróneas en cuanto al aprendizaje y el funcionamiento del cerebro; la necesidad de implementar aprendizajes activos y significativos, que nazcan de situaciones reales de los propios alumnos; la importancia de la memoria y de una vida saludable — sueño, alimentación, ejercicio...-; las ventajas de vivir experiencias tempranas de calidad - cognitivas, sociales y emocionales - como base para un adecuado desarrollo; y, fundamentalmente, la importancia de las emociones en el proceso educativo. Afirman que todo aprendizaje tiene una base emocional, por lo que es urgente atender a la "nutrición emocional” (OCDE, 2007: 95), la gran olvidada en la escuela. Este último aspecto precisa de un replanteamiento en la formación de los docentes. 
El segundo gran desafío al que tendrán que dar respuesta es la creación de una escuela verdaderamente inclusiva. En esa línea, la Asociación Internacional Step by Step (ISSA) afirma que promover el derecho de todos a ser incluidos, respetados y valorados, a participar, trabajar en pro de objetivos comunes y alcanzar su pleno potencial, con atención especial a los más vulnerables (ISSA, 2009), debe convertirse en el objetivo fundamental de la educación. En 1994, la UNESCO y el Ministerio de Educación y Ciencia español, firmaban un documento que serviría de base para desarrollar las políticas sobre Necesidades Educativas Especiales. Se partía del principio universal del derecho a la educación, independiente de las diferencias particulares e instaba al profesorado a formarse para poder dar respuesta a sus necesidades, adaptando los estilos y ritmos de aprendizaje, el curriculum y los recursos pedagógicos (Hegarty, 1994; UNESCO-MEC, 1994).

Años después, nuevamente, la UNESCO (2009a) establece una serie de Directrices sobre politicas de inclusión en la educación. Pese al paso de los años y de las políticas implementadas, se continúa denunciando la exclusión educativa de los niños/as con discapacidad. Según afirman, más de un tercio de ellos no se encuentran escolarizados. Y, en ese sentido, un factor importante para trabajar la inclusión y luchar contra las desigualdades es el papel de los docentes y de los entornos de aprendizaje. "Las escuelas deberían proporcionar a los niños los conocimientos y las competencias necesarias para mantenerse sanos y protegerse del riesgo de ser explotados" (UNESCO, 2009a: 21). Para ello, demandan del docente el conocimiento de las reacciones de los educandos ante el aprendizaje, la implantación de metodologías flexibles y adaptadas, así como la creación de entornos óptimos de aprendizaje (OCDE, 2015). También es necesario el manejo de las nuevas tecnologías adaptadas a la educación y la elaboración de materiales y recursos adecuados. Pero añaden un aspecto esencial en la formación del profesorado: una actitud positiva hacia el aprendizaje y la inclusión. Los docentes deben tener como cualidad personal y profesional la confianza en los educandos, en la capacidad de aprender de todos ellos, independientemente de sus limitaciones.

Las tecnologías de la información y de la comunicación son el tercer gran desafío de los docentes en la sociedad actual. El volumen de información que los alumnos/as pueden encontrar en internet es abrumador, por lo que el profesorado se enfrenta a la dificultad de enseñar a discernir, clasificar, valorar y criticar su validez y autenticidad. Pero tampoco debemos caer en la trampa de considerar las tecnologías, por sí solas, como generadoras de innovación ni de aprendizaje.

Repensar la educación (2015) describe el nuevo escenario de la educación y sus docentes: perder el espacio del aula como lugar único de aprendizaje, incluso "hay quienes sostienen que el modelo de escolaridad no tiene futuro en la era digital a causa de las oportunidades que brindan el aprendizaje electrónico, el aprendizaje móvil y otras tecnologías digitales" (Bokova, 2015a: 50). Esta realidad conduce a la creación de redes de aprendizaje, entendidas como la conexión entre las diferentes instituciones educativas formales y no formales. Por ello, se deben generar sinergias entre los medios sociales, que refuercen los conocimientos y valores aprendidos en la escuela; los dispositivos móviles y recursos educativos que modifican la estructura de clase, las formas de aprender y de buscar la información, transformando el aprendizaje en más informal, personal y ubicuo (Bokova, 2015a); así como los cursos en línea masivos y abiertos que aportan nuevas vías de formación y autoeducación, por lo que "necesariamente tienen que readaptarse y reformularse en función del nuevo contexto sociocultural y tecnológico actual" (UNESCO, 2015: 176) las funciones del docente.

De esta manera, la introducción de las tecnologías de la información y de la comunicación suponen para el profesorado el gran reto de modificar las 
estructuras del aprendizaje, sustituyendo una pedagogía basada en la recepción y la repetición, por otra orientada a "aprender creando", mediante la formación del alumnado como sujeto activo "que reconstruye y da significado a la multitud de información que obtiene extraescolarmente en los múltiples medios de comunicación de la sociedad del siglo XXI, y desarrollar las competencias para utilizar de forma inteligente, crítica y ética la información" (UNESCO, 2015: 182).

Como último reto de la educación y del profesorado, las políticas educativas deben potenciar una adecuada formación de los docentes y una correcta selección de estos. La consultora McKinsey, tras analizar los sistemas educativos de 50 países, llegó a la conclusión de que el nivel educativo de un país depende de la formación inicial, motivación y aprendizaje permanente de sus profesores/as. Dicho en palabras de un funcionario surcoreano la calidad de un sistema educativo tiene como techo la calidad de sus docentes. Esta afirmación parece una obviedad que podría suscribir cualquier funcionario - o cualquier persona sensata - de cualquier país del mundo. Atraer a los estudiantes más brillantes de la enseñanza secundaria a la carrera docente es uno de los rasgos comunes de los países que obtienen mejores resultados en el Informe PISA, como Corea del Sur, Finlandia, Canadá y Hong Kong.

Sin embargo, no en todos los lugares sucede lo mismo. El Informe del comité mixto OIT/UNESCO (2012) identificaba una abundante y sistemática afluencia de docentes no cualificados a los sistemas educativos — debida a la precarización de la profesión-, como uno de los principales obstáculos para la consecución de una educación de calidad. El gran desafío consiste en atraer talentos a la carrera docente en entornos en los que los contratos a corto plazo, la disminución de la remuneración o las condiciones de trabajo no son las más adecuadas. En los países de bajo nivel de renta, los requisitos para ingresar en la profesión seguían siendo escasos y de perfil bajo, no se exigían calificaciones mínimas y en algunos casos se accedía al ejercicio de la docencia con una formación inicial de tres meses.

La creciente necesidad de creatividad y emprendimiento, es decir, la perspectiva económica de la educación, no debe ocultar la necesidad de desarrollar las competencias que los individuos y las comunidades necesitan para los diversos aspectos de la existencia humana. "Se puede afirmar que mantener y aumentar la dignidad, la capacidad y el bienestar de la persona humana en relación con los demás y con la naturaleza debería ser la finalidad fundamental de la educación del siglo XXI” (Bokova, 2015a: 38). Es decir, gestionar de manera eficiente y responsable el ingente volumen de información disponible en el entorno digital y lograr, al mismo tiempo, una edu cación más inclusiva. Dicho de otro modo, una educación que no consista únicamente en la adquisición de aptitudes, sino también en la adquisición de los valores del respeto a la vida y dignidad del ser humano en un mundo caracterizado por la diversidad.

Para ello los profesores siguen constituyendo el recurso más significativo de los sistemas educativos. Tal y como afirma el Informe de la OCDE "el mejorar la eficacia y la equidad de la escolaridad depende, en gran medida, de que pueda garantizarse que los profesores sean personas competentes, que su enseñanza sea de calidad y que todos los estudiantes tengan acceso a una docencia de excelente calidad" (OCDE, 2004: 1). Por lo tanto, es necesario que las políticas educativas de los distintos países sean capaces, en primer lugar, de atraer y retener a los candidatos/as más cualificados a la carrera docente y, en segundo lugar, de consolidar un sistema de formación inicial y continuada que potencie una sólida identidad docente. Por último, debe establecer una carrera profesional que propicie e incentive las buenas prácticas.

El segundo Informe McKinsey (Barber et al., 2012), después de analizar la experiencia de los sistemas educativos de veinte países, puso de manifiesto que los sistemas educativos no conseguirán mejorar 
sus resultados si insisten en continuar haciendo las mismas cosas que tuvieron éxito en el pasado. Tal y como se afirmaba en el Informe de la OCDE (2004), la sociedad demanda de los establecimientos educativos que den cabida a estudiantes de distintas procedencias geográficas, sociales y religiosas; que contemplen las diferencias culturales y de género; que promuevan la convivencia, la cohesión social y la sostenibilidad del entorno; que atiendan adecuadamente a los alumnos/as con problemas de adaptación o aprendizaje y, en definitiva, que sean capaces de preparar a los alumnos/as para seguir desarrollándose en una sociedad marcada por la rapidez e imprevisibilidad de los cambios económicos, sociales, culturales y tecnológicos.

\section{Algunas consideraciones en torno a la formación universitaria de los docentes}

El acierto en las políticas educativas destinadas a clarificar la finalidad de la educación, así como las políticas de formación, selección y promoción del profesorado se van a configurar como la base indispensable para un desarrollo humano, social, político, económico y medioambiental sostenible. Los nuevos desafios que plantea la educación en el siglo XXI precisan de una formación universitaria del profesorado acorde con su importancia y transcendencia, tal y como quedó recogido en la Conferencia Mundial sobre Educación Superior La nueva dinámica de la educación superior y la investigación para el cambio social y el desarrollo (UNESCO, 2009b), que definió entre sus responsabilidades promover el pensamiento crítico y ético entre su estudiantado, amén de desarrollar las competencias profesionales y los conocimientos necesarios para el desempeño profesional. Estas responsabilidades tienen, a nuestro modo de ver, mayor repercusión y alcance en la formación de futuros docentes, encargadas de educar a las nuevas generaciones. En ese sentido, ya en 1995, el Documento de Política para el Cambio y el Desarrollo en la Educación
Superior, ponía el acento en la formación pedagógica del profesorado de educación superior, incluyendo una mención a la importancia de la evaluación "que puede consistir en autoevaluación, evaluación por los pares o evaluación externa" (UNESCO, 1995: 39) como mecanismo fundamental en la política de perfeccionamiento del profesorado de la enseñanza superior. También, en 1998, de nuevo la UNESCO en la Conferencia Mundial sobre Educación Superior de ese año, La Educación Superior en el siglo XXI. Visión y Acción, remarcaba como misión fundamental "contribuir al desarrollo y la mejora de la educación en todos los niveles, en particular mediante la capacitación del personal docente" (UNESCO, 1998: 22). Por tanto, ¿cuál es el rol que deben asumir las Facultades de Educación? ¿Qué enseñanzas y capacidades deben priorizar en su formación universitaria inicial? ¿Cómo adecuar su formación a las competencias básicas que deben adquirir los alumnos? ¿Están las facultades de Educación dando respuesta a estas nuevas demandas?

A la luz de los informes internacionales que hemos ido analizando, emergen una serie de cualidades, de aprendizajes, de competencias que deben ser enseñadas en las diferentes Facultades de Educación y aprendidas por todos los nuevos docentes, si se quiere dar respuesta a los grandes retos que plantea la sociedad del siglo XXI. He aquí algunos de ellas:

- Deben formar personas íntegras, capaces de responder al imperativo moral que demanda la educación (Pérez-Castro, 2011; López Zavala, Solís y Durán, 2013; Sanz, 2014).

- Deben dotar a los futuros docentes de los conocimientos necesarios para implementar entre sus estudiantes las capacidades para vida (Perrenoud, 2012) y las competencias que desarrollen y potencien su empleabilidad.

- Deben convertirse en centros de referencia en innovación y en buenas prácticas en todo lo referente a metodologías docentes, proceso de 
enseñanza-aprendizaje y evaluación educativa. Ser centros de investigación puntera en los nuevos avances y campos de estudio sobre educación, a saber, la neuropedagogía.

- Deben sensibilizar a los alumnos acerca de la importancia del reto que asumen dedicando su vida a la formación y desarrollo de las personas. Potenciar su vocación, su deseo por aprender y por enseñar, así como favorecer la asunción de su rol como compensador de desigualdades, gestor de la diversidad y generador de resiliencia (Day y Gu, 2015).

- Deben dotar de los conocimientos pedagógicos, psicológicos y antropológicos suficientes para enfrentarse a un alumnado cada vez más complejo y diverso, de manera individualizada, personalizada, que atienda a sus diferentes ritmos y estilos de aprendizaje.

- Deben desarrollar entre sus estudiantes la

\section{Referencias}

Aznar, P. y M. A. Ull (2013), La responsabilidad por un mundo sostenible. Propuestas educativas a padres y profesores, Bilbao, Descleé.

Bain, K. (2007), Lo que hacen los mejores profesores universitarios, Valencia, PUV.

Barber, M. y M. Mourshed (2008), Cómo hicieron los sistemas educativos con mejor desempeño en el mundo para alcanzar sus objetivos, Santiago de Chile, Mckinsey.

Barber, M., C. Chijoke y M. Mourshed (2012), Cómo continúan mejorando los sistemas educativos de mayor progreso en el mundo, Santiago de Chile, Mckinsey.

Blakemore, S. J. y U. Frith (2005), Cómo aprende el cerebro. Las claves para la educación, Barcelona, Ariel.

Bokova, I. (2015a), Repensar la educación. ¿Hacia un bien común universal?, París, UNESCO.

Bokova, I. (2015b), Informe de Seguimiento de la Educación para Todos en el Mundo. La educación para todos, 2000-2015. capacidad de innovación, de adaptación a nuevas realidades, en definitiva, convertirse en fuerzas vivas del cambio (Fullan, 2002).

Y todo ello, para dar respuesta a una antigua Recomendación de la OIT-UNESCO (1966: 9) en donde se reclama profesionalidad a los futuros docentes y a sus formadores universitarios:

La enseñanza debería considerarse una profesión cuyos miembros prestan un servicio público; esta profesión exige de los educadores no solamente conocimientos profundos y competencia especial, adquiridos y mantenidos mediante estudios rigurosos y continuos, sino también un sentido de las responsabilidades personales y colectivas que ellos asumen para la educación y el bienestar de los alumnos de que están encargados.

\section{Logros y desafios, París, UNESCO.}

Bokova, I. (2012), Informe de Seguimiento de la Educación para Todos en el Mundo. Los jóvenes y las competencias. Trabajar con la educación, París, UNESCO.

Comisión de las Comunidades Europeas (2007), Comunicación de la Comisión al Consejo y al Parlamento Europeo. Mejorar la calidad de la formación del profesorado, Bruselas.

Day, C. y Q. Gu (2015), Educadores resilientes, escuelas resilientes. Construir y sostener la calidad educativa en tiempos dificiles, Madrid, Narcea.

Delors, J. (1996), La educación encierra un tesoro, Madrid, UNESCO.

Escámez, J. (2013), "El cosmopolitismo y la educación en valores como ciudadano", en M. A. Santos Rego (coord.), Cosmopolitismo y educación. Aprender y trabajar en un mundo sin fronteras, pp. 17-32, Valencia, Brief. 
EURYDICE (2009), Educación y atención a la primera infancia en Europa: un medio para reducir las desigualdades sociales $y$ culturales, Madrid, Ministerio de Educación.

Faure, E. (1973), Aprender a ser. La educación del futuro, París, UNESCO.

Finkel, D. (2008), Dar clase con la boca cerrada, Valencia, PUV. Forés, A. y M. Ligioiz (2009), Descubrir la neurodidáctica. Aprender desde, en y para la vida, Barcelona, Editorial UOC.

Francisco I. (2015), Carta Encíclica 'Laudatio Si' sobre el cuidado de la casa común.

Fullan, M. (2002), Las fuerzas del cambio. Explorando las profundidades de la reforma educativa, Madrid, Akal.

Hargreaves, A. (2000), "Nueva profesionalidad para una profesión paradójica”, en Cuadernos de Pedagogía, núm. 290, pp. 58-60.

Hegarty, S. (1994), Educación de niños y jóvenes con discapacidades, París, UNESCO.

Howard-Jones, P. (2011), Investigación neuroeducativa. Neurociencia, educación y cerebro: de los contextos a la práctica, Madrid, La Muralla.

ISSA (2009), Competent Teachers of the $21^{\text {st }}$ Century, Hungria, International Step by Step Association.

López Zavala, R., M. L. Solís y E. Durán (2013), La docencia como proyecto ético, México, Universidad Autónoma de Sinaloa.

Martín, J., D. Lievesley y R. Kagia (2001), Docentes para las escuelas del mañana. Análisis de los indicadores educativos mundiales, París, OECD-UNESCO.

Matsuura, K. (2008), Informe de Seguimiento de la Educación para Todos en el Mundo. Educación para Todos en 2015. ¿Alcanzaremos la meta?, París, UNESCO.

Mora, F. (2015), Neuroeducación. Solo se puede aprender aquello que se ama, Madrid, Alianza.

Noddings, N. (2002), Educating moral people: a caring alternative to caracter education, Nueva York, Teachers College Press.

Nussbaum, M. (2013), Crear capacidades. Propuesta para el desarrollo humano, Barcelona, Paidós.

Nussbaum, M. (2010), Sin fines de lucro. Por qué la democracia necesita de las Humanidades, Buenos Aires, Katz.

Nussbaum, M. (1999), Los límites del patriotismo. Identidad, pertenencia y "ciudadanía mundial", Barcelona, Paidós.
Nussbaum, M. y A. Sen (comp.) (1996), La calidad de vida, México, Fondo de Cultura Económica.

Observatorio de Innovación Educativa del Tecnológico de Monterrey (2015), Aprendizaje basado en Retos, Monterrey, Tecnológico de Monterrey.

OCDE (2015), Politica educativa en perspectiva 2015. Hacer posibles las reformas, Madrid, Fundación Santillana.

OCDE (2007), La comprensión del cerebro. El nacimiento de una ciencia del aprendizaje, París, Ediciones Universidad Católica Silva Henríquez.

OCDE (2004), La cuestión del profesorado: atraer, capacitar y conservar profesores eficientes, París, OECD, Rights and Traslation Unit.

OCDE (2001), Education Policy Analysis, París.

OCDE-UNESCO (2001), Docentes para las escuelas del mañana. Análisis de los indicadores educativos mundiales, París, OCDEUNESCO.

OEI (2014), Miradas sobre la educación en Iberoamérica. Avances en las Metas Educativas 2021, Madrid, OEI.

OIT/UNESCO (2012), Informe final Comité Mixto OIT/ UNESCO de expertos sobre la aplicación de las recomendaciones relativas al personal docente, Ginebra, Organización Internacional del Trabajo.

OIT/UNESCO (1966), Recomendación conjunta de la OIT/ UNESCO relativa a la situación del personal docente, Ginebra, Organización Internacional del Trabajo.

ONU (2015), Objetivos de desarrollo del milenio. Informe de 2015, Nueva York, Naciones Unidas.

Pérez-Castro, J. (2011), "Los valores universitarios ante los cambios en el sistema educativo," en A. Hirsch y R. López Zavala (coords.), Ética y valores profesionales. Trece experiencias de investigación universitaria en México, México, Universidad Autónoma de Sinaloa, pp. 263-290.

Perrenoud, P. (2012), Cuando la escuela pretende preparar para la vida. ¿Desarrollar competencias o enseñar otros saberes?, Barcelona, Graó.

Pizarro, B. (2003), Neurociencia y educación, Madrid, La Muralla.

PNUD (1999), Informe sobre desarrollo humano 1999: la mundialización con rostro humano, Nueva York, Oxford University Press. 
PNUD (2000), Informe sobre desarrollo humano 2000: derechos humanos y desarrollo humano, Nueva York, Oxford University Press.

PNUD (2004), Informe sobre desarrollo humano 2004: la libertad cultural en el mundo diverso de hoy, Nueva York, Oxford University Press.

Sanz, R. (2014), "La necesidad de una formación ética en la educación inicial de los alumnos del Magisterio," en A. Hirsch y R. López Zavala (coords.). Ética profesional en Educación Superior. Finalidades, estrategias y desafios de la formación, México, Universidad Autónoma de Sinaloa, pp. 79-99.

Stenhouse, L. (1988), "Artistry and Teaching: the teacher as focus of research and development", en Gournal of Curriculum and Supervision, vol. 4, núm. 1, pp. 43-51.

UNESCO (2015), Mejorar aprendizajes en la educación obligatoria. Políticas y actores, Buenos Aires, UNESCO.

UNESCO (2012), Oportunidades perdidas: el impacto de la repetición y de la salida prematura de la escuela, Montreal, UNESCO.
UNESCO (2009a), Directrices sobre políticas de inclusión en la educación, París, UNESCO.

UNESCO (2009b), Conferencia Mundial sobre la Educación Superior. La nueva dinámica de la Educación Superior y la investigación para el cambio social y el desarrollo, París, UNESCO.

UNESCO (1998), Conferencia Mundial sobre la Educación Superior. La Educación Superior en el siglo XXI. Visión y Acción, París, UNESCO.

UNESGO (1995), Documento de Política para el Cambio y el Desarrollo en la Educación Superior, París, UNESCO.

UNESCO-CUE (2013a), "Hacia un aprendizaje universal. Lo que cada niño debería aprender", Resumen ejecutivo. Informe $n^{\circ} 1$ de la Comisión especial sobre métricas de los aprendizajes, UNESCO.

UNESCO-CUE (2013b), "Hacia un aprendizaje universal. Recomendaciones de la comisión especial sobre métricas de los aprendizajes", UNESCO.

UNESCO-MEG (1994), Declaración de Salamanca y Marco Acción para las Necesidades Educativas Especiales, Salamanca, UNESCO.

Cómo citar este artículo:

Sanz-Ponce, Roberto y Aurelio González-Bertolín (2018), "La educación sigue siendo un "tesoro". Educación y docentes en los informes internacionales de la UNESCO", en Revista Iberoamericana de Educación Superior (RIES), México, UNAM-IISUE/Universia, vol. IX, núm. 25, pp. 157-174, http://dx.doi.org/10.22201/ iisue.20072872e.2019.25.347 [consulta: fecha de última consulta]. 